\title{
An Arthroscopic Device to Assess Articular Cartilage Defects and Treatment with a Hydrogel
}

\author{
William J. McCarty, ${ }^{1}$ Anna Luan, ${ }^{1}$ Priya Sundaramurthy, ${ }^{1}$ Caryn Urbanczyk, ${ }^{1}$ Atal Patel, ${ }^{1}$ \\ Jacob Hahr, ${ }^{1}$ Mohammad Sotoudeh, ${ }^{2}$ Anthony Ratcliffe, ${ }^{2}$ and Robert L. Sah ${ }^{1,3}$ \\ ${ }^{1}$ Department of Bioengineering, University of California-San Diego, 9500 Gilman Drive, Mail Code 0412, La Jolla, \\ CA 92093-0412, USA; ${ }^{2}$ Synthasome, Inc., La Jolla, CA, USA; and ${ }^{3}$ Institute of Engineering in Medicine, University \\ of California-San Diego, La Jolla, CA, USA
}

(Received 18 July 2010; accepted 12 November 2010; published online 24 November 2010)

Associate Editor Angelique Louie oversaw the review of this article.

\begin{abstract}
The hydraulic resistance $R$ across osteochondral tissue, especially articular cartilage, decreases with degeneration and erosion. Clinically useful measures to quantify and diagnose the extent of cartilage degeneration and efficacy of repair strategies, especially with regard to pressure maintenance, are still developing. The hypothesis of this study was that hydraulic resistance provides a quantitative measure of osteochondral tissue that could be used to evaluate the state of cartilage damage and repair. The aims were to (1) develop a device to measure $R$ in an arthroscopic setting, (2) determine whether the device could detect differences in $R$ for cartilage, an osteochondral defect, and cartilage treated using a hydrogel ex vivo, and (3) determine how quickly such differences could be discerned. The apparent hydraulic resistance of defect samples was $\sim 35 \%$ less than intact cartilage controls, while the resistance of hydrogel-filled groups was not statistically different than controls, suggesting some restoration of fluid pressurization in the defect region by the hydrogel. Differences in hydraulic resistance between control and defect groups were apparent after $4 \mathrm{~s}$. The results indicate that the measurement of $R$ is feasible for rapid and quantitative functional assessment of the extent of osteochondral defects and repair. The arthroscopic compatibility of the device demonstrates the potential for this measurement to be made in a clinical setting.
\end{abstract}

Keywords-Hydraulic resistance, Arthroscopic device, Osteochondral defect, Hydrogel.

\section{INTRODUCTION}

Articular cartilage is a load-bearing connective tissue at the ends of long bones in synovial joints that facilitates low-friction, low-wear joint articulation. The load-bearing ability of cartilage is dependent on the

Address correspondence to Robert L. Sah, Department of Bioengineering, University of California-San Diego, 9500 Gilman Drive, Mail Code 0412, La Jolla, CA 92093-0412, USA. Electronic mail:rsah@ucsd.edu presence of a large aggregating proteoglycan, aggrecan, in the matrix. ${ }^{4}$ Aggrecan is highly negatively charged due to its numerous sulfated glycosaminoglycan (GAG) side chains, and the charge density of these sulfated GAG moieties creates a swelling pressure in the interstitial fluid of cartilage that resists compression. ${ }^{18}$ The permeability, or ease of fluid flow through cartilage, is inversely related to the tissue charge density, ${ }^{17}$ and is low in normal tissue,${ }^{6}$ which is essential for fluid pressurization. Interstitial fluid pressurization supports most of the load that cartilage receives, shielding the solid matrix from excessive strain. ${ }^{26}$

While permeability has traditionally been reported for individual materials, such as articular cartilage, related variables such as hydraulic resistance are needed to describe complex multi-tissue structures. Permeability, or specific permeability, typically refers to the constant $K$ in Darcy's empirical law. ${ }^{15,27}$ Hydraulic resistance $R$ is similar, though inversely related to permeability

$$
\begin{aligned}
& R=\left(\frac{\Delta P}{Q}\right), \quad K=\frac{\mu L}{A}\left(\frac{Q}{\Delta P}\right), \\
& K^{\prime}=k_{\mathrm{p}}=\frac{Q / A}{\Delta P / L}=\frac{L}{A}\left(\frac{Q}{\Delta P}\right), \\
& K^{\prime \prime}=c=\frac{1}{A}\left(\frac{Q}{\Delta P}\right),
\end{aligned}
$$

with viscosity $\mu$, flow rate $Q$, fluid path-length $L$, pressure $P$, and cross-sectional area $A$. Less specific forms of permeability have been defined, including hydraulic permeability $k_{\mathrm{p}}$ or hydraulic conductivity $K^{\prime}$, and hydraulic conductance $c$. The use of the less specific definitions, $c$ or $R$, is appropriate when multi-tissue structures are used, such as osteochondral tissue, ${ }^{13}$ as $K$ and $k_{\mathrm{p}}$ are typically defined for individual materials. 
The hydraulic resistance across osteochondral tissue, especially articular cartilage, decreases with degeneration and erosion. Progressive degeneration and erosion of articular cartilage that can occur with osteoarthritis $(\mathrm{OA})$ has been correlated with decreased hydraulic resistance. ${ }^{1}$ In addition, focal defects, which are commonly observed in the knees of symptomatic patients during arthroscopy, ${ }^{8,12}$ are discrete areas of cartilage erosion that also likely have reduced hydraulic resistance. Differences in the hydraulic resistance of osteochondral tissue with normal, partially, and fully eroded cartilage have also been reported. ${ }^{13}$ Such decreases in hydraulic resistance will diminish the ability of cartilage to maintain fluid pressurization, leading to larger strains on the cartilage matrix and likely to further degeneration. ${ }^{18,30,31}$

Different repair strategies for cartilage defects are being investigated, though only a limited number address the need for immediate restoration of interstitial fluid pressurization. Defect repair strategies include arthroscopic procedures, such as microfracture ${ }^{25}$; soft tissue grafts ${ }^{5}$; osteochondral grafts of autogenic ${ }^{11}$ or allogenic source material ${ }^{9}$; cell transplantation with or without a scaffold, including autologous cell implantation $^{3}$ and mesenchymal stem cells; and synthetic and natural scaffolds. Interstitial fluid pressurization, and load-bearing capacity, is typically restored with the osteochondral graft techniques, and for certain scaffold implants, such as high concentration formulations of poly-(ethylene glycol) with high stiffness (similar to cartilage stiffness) and low permeability, ${ }^{16,23}$ while most cell-based techniques make use of a scaffold that is initially less stiff than normal cartilage.

Clinically useful measures to diagnose the extent of cartilage degeneration and efficacy of repair strategies are limited, especially with regard to pressure maintenance. Imaging modalities have typically been used to diagnose cartilage degeneration and defects, including plain film X-ray attenuation, magnetic resonance imaging (MRI), computed tomography (CT), and visual observation during arthroscopy. Though these methods alone have limited quantitative ability, contrast-enhanced MRI, used clinically, ${ }^{14}$ and CT, used in small animal models, ${ }^{22,32}$ can allow for the determination of spatial maps of charge density in cartilage, an indirect measure of hydraulic resistance, though the resolution of MRI techniques widely available clinically lags behind what is reported in academia. Cartilage degeneration detection using optical coherence tomography has also recently been reported. ${ }^{7}$ Arthroscopic instruments for the determination of stress-strain behavior or electrical impedance, from which hydraulic resistance could be inferred, have been suggested, ${ }^{2,21,28}$ but are not yet in clinical practice in the United States. A device allowing the direct perfusion-based measurement of hydraulic resistance in an arthroscopic setting has not yet been described.

Thus, the hypothesis of this study was that hydraulic resistance provides a quantitative measure of osteochondral tissue that could be used to evaluate the state of cartilage damage and treatment. The aims were to (1) develop a device to assess hydraulic resistance in an arthroscopic setting, (2) determine whether the device could detect differences in hydraulic resistance for cartilage, an osteochondral defect, and a defect treated with a low permeability hydrogel, and (3) determine how quickly such differences could be discerned.

\section{METHODS}

\section{Study Design}

An arthroscopic probe was used to measure the hydraulic resistance of osteochondral tissue ex vivo. Ten osteochondral blocks were harvested from the medial and lateral femoral condyles of five adult bovine knees, with 1 block per condyle. Samples were wrapped in gauze soaked with PBS with protease inhibitors (PBS + PI: $1 \mathrm{mM}$ phenylmethanesulfonyl fluoride, $2 \mathrm{mM}$ disodium ethylenediamine tetraacetate, $5 \mathrm{mM}$ benzamidine- $\mathrm{HCl}$, and $10 \mathrm{mM} N$-ethylmaleimide) and stored at $-20{ }^{\circ} \mathrm{C}$ until testing. The hydraulic resistance of each block was tested in a single location on the load-bearing, posterior face of the condyle on (1) intact cartilage, (2) after drilling an osteochondral defect, and (3) after filling the defect with a photopolymerized hydrogel.

\section{Arthroscopic Probe Device Design and Testing}

A prototype for a potentially arthroscopically deliverable probe tip that sealed against normal cartilage or the cartilage surface surrounding a defect to allow the measurement of hydraulic resistance was fabricated and calibrated. The probe consisted of a cylindrical silicone rubber cap $(8 \mathrm{~mm}$ height by $15 \mathrm{~mm}$ inner diameter by $1.4 \mathrm{~mm}$ thick) glued on a flat silicone rubber donut (18 $\mathrm{mm}$ outer, $10 \mathrm{~mm}$ inner diameter by $1.0 \mathrm{~mm}$ thick) with a silicone rubber disc $(23 \mathrm{~mm}$ diameter by $2.0 \mathrm{~mm}$ thick) glued to the closed end. A 3-mm diameter hole was punched in the side of the cap, and a stainless steel tube $(4.5 \mathrm{~mm}$ outer diameter by $0.13 \mathrm{~mm}$ thick) adhered inside silicone tubing (3.8 $\mathrm{mm}$ inner diameter by $1.0 \mathrm{~mm}$ thick) was secured to this hole, creating a single compartment space (Fig. 1). The range of pressures the probe could withstand without collapse was tested against intact cartilage, and the probe dimensions were tested by passing it through an $8-\mathrm{mm}$ diameter stainless steel tube to 


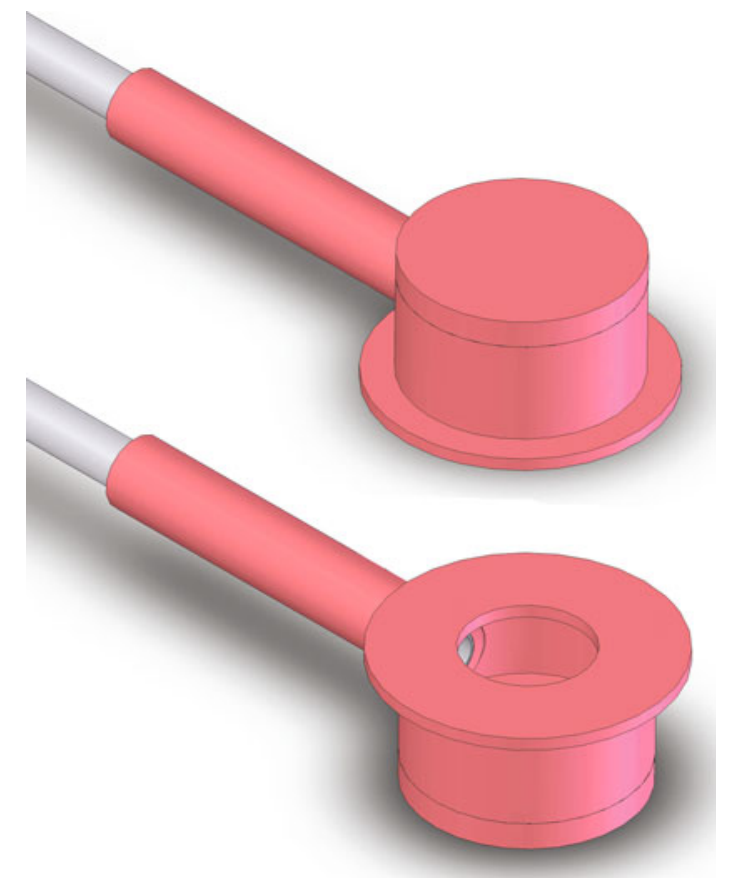

FIGURE 1. Schematic representation of the arthroscopic probe device in an isometric view from the top and bottom.

simulate a standard arthroscopic cannula (typical inner diameter range of 4.5-8 $\mathrm{mm}$ ).

Using this device, $R$ was measured. A perfusion apparatus consisting of a syringe pump and pressure transducer was assembled and calibrated. A $20-\mathrm{mL}$ plastic syringe was filled with phosphate buffered saline (PBS) and placed in a syringe pump (Harvard PHD 2000; Harvard Apparatus, Holliston, MA). The syringe was connected to the stainless steel tube of the probe device and to a pressure sensor (DP15 Low Pressure Sensor; Validyne Engineering, Northridge, CA) using stiff tubing. The pressure sensor was calibrated to confirm linearity between 0 and $344 \mathrm{kPa}$.

The hydraulic resistance of each sample with intact cartilage, after creation of a defect and after hydrogel polymerization, was measured using the device and setup described. The tissue blocks and device were submerged in a bath of PBS. The device was placed against the sample surface, held in place with a clamp, a weight $(\sim 0.5 \mathrm{~N}$ normal force) was placed on top of the probe tip, and the pressure was recorded for $20 \mathrm{~s}$ without flow, and then for $40 \mathrm{~s}$ after the flow was initiated at a rate of $167 \mathrm{~mm}^{3} \mathrm{~s}^{-1}$. Once the flow was initiated, the negative pressure generated inside the probe tip was sufficient to maintain the probe position against the sample surface, and the normal force was removed. After testing the intact samples, $10 \mathrm{~mm}$ diameter by $4 \mathrm{~mm}$ deep defects were created through the cartilage and into the subchondral bone, though not into the cancellous bone, using a drill press while irrigating with PBS, and retested. The device was placed directly over the defect, enclosing it within the probe tip such that the outer edge of the probe tip was completely surrounding the defect site and the inner edge was within the defect region. Finally, the defects were filled with poly-(ethylene glycol) diacrylate hydrogel $(30 \% \mathrm{w} / \mathrm{v} \text {, Synthasome Inc, La Jolla, CA })^{23}$ that was adhered to the surrounding articular cartilage and bone by tissue initiated photopolymerization ${ }^{29}$ using an A4000 series UV light source (EXFO, Mississauga, Canada), and samples retested. Each sample was tested three times in each condition. The hydraulic permeability of $3 \mathrm{~mm}$ thick samples of hydrogel was assessed in vitro by direct perfusion as $3.4 \pm 0.8 \times 10^{-14}\left[\mathrm{~m}^{2} \mathrm{~Pa}^{-1} \mathrm{~s}^{-1}\right](n=3)$, slightly more permeable than the range of $1 \times 10^{-16}$ to $2 \times 10^{-15}$ $\left[\mathrm{m}^{2} \mathrm{~Pa}^{-1} \mathrm{~s}^{-1}\right]$ reported for cartilage, ${ }^{17,19,20}$ and much less permeable than subchondral bone $\sim 1 \times 10^{-10}$ $\left[\mathrm{m}^{2} \mathrm{~Pa}^{-1} \mathrm{~s}^{-1}\right] .^{13}$

\section{Data Reduction and Curve Fitting}

Experimental data were reduced and fit to a model equation to determine the hydraulic resistance and compliance parameters. For each trial, pressure values were normalized to the average pressure recorded over $5 \mathrm{~s}$ before initiation of flow. The zero time point was set as the point where the pressure increased over 3 standard deviations from the baseline average. The three trials for each condition of pressure vs. time data were averaged at each time point. The experimental setup was modeled as a parallel $R C$ lumped parameter circuit with a current source (Fig. 2), assuming the hydraulic resistance through the sample was the dominant resistance $R\left[\mathrm{kPa} \mathrm{s} \mathrm{mm}^{-3}\right]$, the total system compliance $C\left[\mathrm{~mm}^{3} \mathrm{kPa}^{-1}\right]$, flow rate $Q\left[\mathrm{~mm}^{3} \mathrm{~s}^{-1}\right]$, pressure $P[\mathrm{kPa}]$, and time $t[\mathrm{~s}]$. Averaged $P$ vs. time data sets were fit to the solution equation

$$
(-P)=Q R\left(1-\exp \left[-\frac{t}{R \cdot C}\right]\right)
$$

to estimate $R$ for each trial and a single, global $C$ value using an ordinary least square error nonlinear regression (Excel 2003, Microsoft Corporation, Redmond, WA).

\section{Statistical Analysis}

Data are presented as mean \pm SEM for $n=10$ samples. A 2-way repeated measures ANOVA was used to determine the effects of tissue condition (intact, defect, hydrogel) and time ( $t=1,2,4,8,16,32 \mathrm{~s})$ on measured pressure. A 1-way ANOVA was used to determine the fixed effect of condition (intact, defect, hydrogel) on hydraulic resistance. When significant 


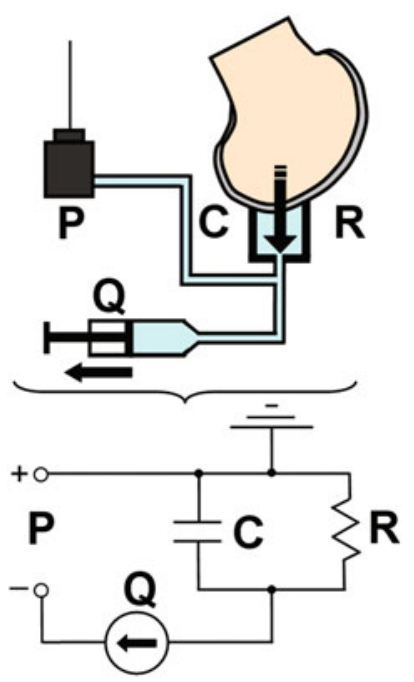

FIGURE 2. The experimental setup was modeled as a parallel RC lumped parameter circuit with hydraulic resistance $R$, the total system compliance $C$, flow rate $Q$, and pressure $P$.

effects were determined, $p<0.05$, differences between groups were assessed with Tukey post hoc tests.

\section{RESULTS}

The arthroscopic probe device sealed against the cartilage surface upon initiation of flow and the resulting pressures were measured for samples with intact cartilage, defects, and hydrogel-filled defects. The device was able to seal against the cartilage surface and maintain pressures over $344 \mathrm{kPa}$. The lateral walls and top of the cylindrical silicone rubber cap deformed for $\sim 5 \mathrm{~s}$ with increasing pressure, resulting in an approximately $15 \%$ decrease in cap volume before stabilization.

The pressure vs. time data revealed differences between the pressure responses from samples with intact cartilage, defects, and hydrogel-filled defects. Pressure varied with cartilage condition (intact, defect, or hydrogel; $p<0.001$ ) and time after initiation of flow $(p<0.0001)$, with an interaction effect $(p<0.0001)$. Differences in pressure between the intact and defect groups were significant at all times from 4 to $32 \mathrm{~s}$ $(p<0.0001-0.05$; Fig. 3 ). The pressure response from the hydrogel-filled defects was not statistically different than the unfilled defects at 4 and $8 \mathrm{~s}$, but was different than the unfilled defects, and not different than the intact cartilage, at 16 and $32 \mathrm{~s}$.

The best-fit parameters for hydraulic resistance also showed differences between the intact cartilage, defects, and hydrogel-filled defects. $R$ varied significantly with cartilage condition $(p<0.00001$, Fig. 4), with the best-fit value for system compliance of $26.4 \mathrm{~mm}^{3} \mathrm{kPa}^{-1}$. On average, $R_{\text {defect }}$ values were $35 \%$

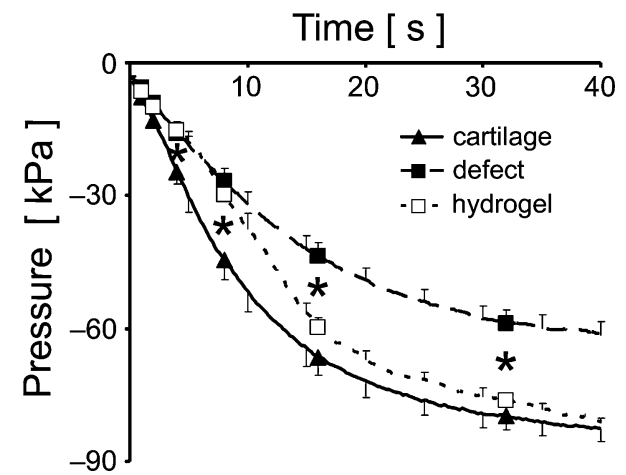

FIGURE 3. Pressure vs. time curves for intact cartilage, osteochondral defects, and defects filled with hydrogel, $n=10$. Triangle and square markers indicate the $1,2,4,8,16$, and $32 \mathrm{~s}$ time points that were analyzed. * $p<0.05$ difference between groups above and below the asterisk.

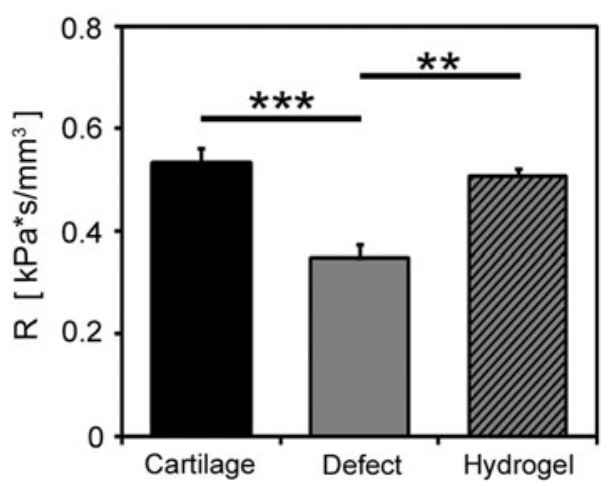

FIGURE 4. Best-fit values of resistance $R$ at a compliance $C$ of $26.4 \mathrm{~mm}^{3} \mathrm{kPa}^{-1}$ for intact cartilage, osteochondral defects, and defects filled with hydrogel, $n=10 .{ }^{* *} p<0.001$, ${ }^{\star \star \star} p<0.0001$.

less than $R_{\text {cartilage values }(p<0.0001) \text { and } 31 \% \text { less }}$ than $R_{\text {gel }}$ values $(p<0.001)$, while $R_{\text {gel }}$ values were not significantly different than $R_{\text {cartilage values }}(p=0.75)$.

\section{DISCUSSION}

The results indicate that the measurement of hydraulic resistance is feasible for rapid and quantitative functional assessment of osteochondral defects and treatments. The magnitude of pressure generated against the samples with an intact cartilage surface was significantly higher than that generated against the samples with an osteochondral defect (Fig. 3), indicating a loss of hydraulic resistance (Fig. 4) that was apparent after a few seconds. The application of the hydrogel to the defects increased the magnitude of pressure generated compared to the defect samples at longer times (Fig. 3), while the hydraulic resistance value was increased to levels similar to those measured for intact cartilage (Fig. 4). 
The differences in hydraulic resistance measured between conditions are a proof of concept using a prototype device for the idea of an arthroscopic probe to quantify local regions of cartilage degeneration and abnormal fluid communication between the intraarticular space and bone. The defects created were severe, such as could be found in intra-articular fractures or significantly advanced OA with wear of the subchondral bone, where the defects are visible. Additional experiments could be performed to determine whether finer differences in hydraulic resistance can be assessed, such as in normal osteochondral tissue depleted of GAG and osteoarthritic tissue with sites of grade II or III degeneration (Modified Outterbridge Scale), using the current device, or after varying device design parameters to increase the resolution.

The ability of the device to resolve finer differences, including cartilage fibrillation or partial- or full-thickness erosion, on a reasonable time-scale depends on several device and setup properties, including stiffness of the probe material and volume of the chamber. The system compliance and sample resistance determine the sensitivity of the probe and time constant, and, along with the flow rate, the pressure magnitudes obtained. A lower system compliance would increase the sensitivity of the device, as a smaller deformation of the stiffer probe tip would increases the fluid pressure generated. In addition, the increase in fluid flow due to degenerate or eroded cartilage compared to healthy cartilage may be masked by flow associated with the system compliance. The current compliance of $\sim 26 \mathrm{~mm}^{3} \mathrm{kPa}^{-1}$ could be significantly reduced by increasing the stiffness of the probe material and decreasing the probe chamber volume, as well as by reducing any compliance in the pressure sensor and syringe, increasing the sensitivity of the device. Thus, additional work could be done to increase the sensitivity of the device and to characterize the ability of the probe to reveal partial defects.

The hydraulic resistance values measured here are apparent values for osteochondral tissue that could be useful to determine the extent of cartilage erosion. While the values of hydraulic resistance measured using this device are several orders of magnitude less than those that could be calculated for articular cartilage alone based on previously reported hydraulic permeability values from direct perfusion measurements, ${ }^{17,19,20}$ the comparison between intact and damaged cartilage, including assessing whether a fluidic short circuit through intact cartilage or any defect treatment implant exists, is the intended purpose of the device. Determining a resistance value similar to cartilage indicates that there is some fluid pressurization in the defect area and that there is minimal abnormal fluid flow in that region between the intraarticular space and the bone. Direct perfusion experiments to determine the hydraulic permeability of cartilage are technically difficult, requiring tens of hours for fluid pressurization before running time and controls for tissue deformation with pressure among other challenges, making their clinical utility limited. By determining the apparent hydraulic resistance, this device and measurement procedure were able to determine differences between intact cartilage and defects in tens of seconds, instead of hours.

The arthroscopic compatibility of the device design and short time constant demonstrate the potential for this measurement to be made in a clinically useful setting. The design of the actual instrument for this purpose, instead of the prototype described here, would need to have a method for ensuring no portion of the device could detach and become a loose body in the joint and would be tested using standard arthroscopic tools The device materials were chosen such that the cap portion could be temporarily deformed to pass through a standard arthroscopy cannula with an 8-mm inner diameter, allowing for its use in an arthroscopic setting. The time constant $\tau$

$$
\tau=R \cdot C
$$

is a reasonable estimate of the time over which pressure would need to be collected in order to have data covering a significant portion of the pressure curve. The time constants for determination of the pressure curve averaged $14.1 \pm 0.8 \mathrm{~s}$ for intact cartilage, $9.2 \pm 0.7 \mathrm{~s}$ for cartilage with a defect, and $13.4 \pm 0.3 \mathrm{~s}$ for a defect filled with hydrogel. Obtaining data beyond the initial few seconds, at which point absolute differences in pressure were already significantly different between intact and defect samples (Fig. 3), is necessary to fit the data to the model equation, as the initial slope at $t=0$ is independent of $R$, as shown by taking the derivative of Eq. (5) with respect to time

$$
\left.\frac{d(-P)}{d t}\right|_{t=0}=\frac{Q}{C}
$$

With only approximately $20 \mathrm{~s}$ necessary for data collection ( $\sim 5 \mathrm{~s}$ of no flow and $\sim 15 \mathrm{~s}$ with flow), and negative pressure holding the device in place after a brief push, the time that would be added to an arthroscopic surgery to obtain a quantitative measurement of damage to a local region of cartilage is a reasonable tradeoff.

The increased hydraulic resistance following application of the hydrogel suggests there was some restoration of fluid pressurization function at the defect area. The shape of the pressure curve for the hydrogel samples suggests that there is more compliance in the hydrogel than in cartilage, as the pressures generated were different in magnitude from intact samples and similar to the defect samples up until $8 \mathrm{~s}$ (Fig. 3). After that time, 
the pressures transitioned to become similar to the intact samples, with significantly higher hydraulic resistance than the defect samples, indicating that some hydraulic resistance had been restored to the defect. Cartilage repair strategies that restore the fluid pressurization, such as the high concentration formulation of photopolymerized PEG hydrogel with high stiffness and low permeability used here, have the advantage of allowing the load-bearing functions of the tissue to resume, while reducing the exaggerated loads on the surrounding tissue that occur with regions of low stiffness.

Further experimentation could be performed to determine the effects of the negative pressure that is generated on the cartilage and bone. Articular cartilage normally experiences fluid pressurization during loading, with estimates of physiological magnitudes of pressure up to $20 \mathrm{MPa},{ }^{24}$ depending on joint, type of activity, body weight, and location, among other factors, which is about 50 times higher than the pressures used here. Positive hydrostatic pressures of $2.5 \mathrm{MPa}$ applied for $20 \mathrm{~s}$ stimulate matrix synthetic rates of chondrocytes ${ }^{10}$ indicating that even short procedures may affect chondrocyte metabolism, though negative pressures may have different effects than hydrostatic pressure. Investigating whether chondrocyte viability is altered by the portion of the device that seals to the cartilage surface would also be important.

The results of this study demonstrate the potential use of measuring hydraulic resistance as a diagnostic tool and describe a practical device that could be used to make such measurements. While this data provide a proof of principle, additional work determining how fine of degradative changes can be discerned and characterizing the effects of a similar procedure on bone and cartilage in vivo could be performed. Such a device allows for a quantitative assessment of the fluid pressurization ability of articular cartilage that can be used in the arthroscopic setting to complement and extend current diagnostic tools.

\section{ACKNOWLEDGMENTS}

This work was supported by grants from the National Institutes of Health and an award to UCSD from the Howard Hughes Medical Institute through the HHMI Professors Program (for RLS). The authors would like to thank the UCSD Senior Design course BENG 187.

\section{OPEN ACCESS}

This article is distributed under the terms of the Creative Commons Attribution Noncommercial
License which permits any noncommercial use, distribution, and reproduction in any medium, provided the original author(s) and source are credited.

\section{REFERENCES}

${ }^{1}$ Armstrong, C. G., and V. C. Mow. Variations in the intrinsic mechanical properties of human articular cartilage with age, degeneration, and water content. J. Bone Joint Surg. Am. 64-A:88-94, 1982.

${ }^{2}$ Athanasiou, K., and G. Constantinides. Arthroscopic indenter. U.S. Patent \#5433215, Board of Regents, University of Texas System, 1995.

${ }^{3}$ Brittberg, M., A. Lindahl, A. Nilsson, C. Ohlsson, O. Isaksson, and L. Peterson. Treatment of deep cartilage defects in the knee with autologous chondrocyte transplantation. N. Engl. J. Med. 331:889-895, 1994.

${ }^{4}$ Buckwalter, J. A., and H. J. Mankin. Articular cartilage. Part I: tissue design and chondrocyte-matrix interactions. J. Bone Joint Surg. Am. 79-A:600-611, 1997.

${ }^{5}$ Carranza-Bencano, A., L. Garcia-Paino, J. R. Armas Padron, and A. Cayuela Dominguez. Neochondrogenesis in repair of full-thickness articular cartilage defects using free autogenous periosteal grafts in the rabbit. A follow-up in six months. Osteoarthr. Cartil. 8:351-358, 2000.

${ }^{6}$ Chen, A. C., W. C. Bae, R. M. Schinagl, and R. L. Sah. Depth- and strain-dependent mechanical and electromechanical properties of full-thickness bovine articular cartilage in confined compression. J. Biomech. 34:1-12, 2001.

${ }^{7}$ Chu, C. R., A. Williams, D. Tolliver, C. K. Kwoh, S. Bruno, III, and J. J. Irrgang. Clinical optical coherence tomography of early articular cartilage degeneration in patients with degenerative meniscal tears. Arthritis Rheum. 62:1412-1420, 2010.

${ }^{8}$ Curl, W. W., J. Krome, E. S. Gordon, J. Rushing, B. P. Smith, and G. G. Poehling. Cartilage injuries: a review of 31,516 knee arthroscopies. Arthroscopy 13:456460, 1997.

${ }^{9}$ Gortz, S., and W. D. Bugbee. Allografts in articular cartilage repair. J. Bone Joint Surg. Am. 88:1374-1384, 2006.

${ }^{10}$ Hall, A. C., J. P. G. Urban, and K. A. Gehl. The effects of hydrostatic pressure on matrix synthesis in articular cartilage. J. Orthop. Res. 9:1-10, 1991.

${ }^{11}$ Hangody, L., G. Kish, Z. Karpati, I. Szerb, and I. Udvarhelyi. Arthroscopic autogenous osteochondral mosaicplasty for the treatment of femoral condylar articular defects. A preliminary report. Knee Surg. Sports Traumatol. Arthrosc. 5:262-267, 1997.

${ }^{12}$ Hjelle, K., E. Solheim, T. Strand, R. Muri, and M. Brittberg. Articular cartilage defects in 1,000 knee arthroscopies. Arthroscopy 18:730-734, 2002.

${ }^{13}$ Hwang, J., W. C. Bae, W. Shieu, C. W. Lewis, W. D. Bugbee, and R. L. Sah. Increased hydraulic conductance of human articular cartilage and subchondral bone plate with progression of osteoarthritis. Arthritis Rheum. 58:38313842, 2008.

${ }^{14}$ Kim, Y. J., D. Jaramillo, M. B. Millis, M. L. Gray, and D. Burstein. Assessment of early osteoarthritis in hip dysplasia with delayed gadolinium-enhanced magnetic resonance imaging of cartilage. J. Bone Joint Surg. Am. 85-A:1987-1992, 2003. 
${ }^{15}$ Levick, J. R. Flow through interstitium and other fibrous matrices. Q. J. Exp. Physiol. 72:409-437, 1987.

${ }^{16}$ Li, Q., J. Wang, S. Shahani, D. D. Sun, B. Sharma, J. H. Elisseeff, and K. W. Leong. Biodegradable and photocrosslinkable polyphosphoester hydrogel. Biomaterials 27:1027-1034, 2006.

${ }^{17}$ Maroudas, A. Physicochemical properties of cartilage in the light of ion exchange theory. Biophys. J. 8:575-595, 1968.

${ }^{18}$ Maroudas, A. Balance between swelling pressure and collagen tension in normal and degenerate cartilage. Nature 260:808-809, 1976.

${ }^{19}$ Maroudas, A., and P. Bullough. Permeability of articular cartilage. Nature 219:1260-1261, 1968.

${ }^{20} \mathrm{McCutchen}, \mathrm{C}$. W. The frictional properties of animal joints. Wear 5:1-17, 1962.

${ }^{21}$ McPherson, R., N. Shrive, E. Damson, C. Frank, F. Lhenen, and N. Schachar. Tissue softness probe. U.S. Patent \#5701913, University Technologies International Inc., 1997.

${ }^{22}$ Palmer, A. W., R. E. Guldberg, and M. E. Levenston. Analysis of cartilage matrix fixed charge density and threedimensional morphology via contrast-enhanced microcomputed tomography. Proc. Natl. Acad. Sci. USA 103:19255-19260, 2006.

${ }^{23}$ Riley, S. L., S. Dutt, R. de la Torre, A. C. Chen, R. L. Sah, and A. Ratcliffe. Formulation of PEG-based hydrogels affects tissue-engineered cartilage construct characteristics. J. Mater. Sci. Mater. Med. 12:983-990, 2001.

${ }^{24}$ Rushfeldt, P. D., R. W. Mann, and W. H. Harris. Improved techniques for measuring in vitro the geometry and pressure distribution in the human acetabulum. I. Ultrasonic measurement of acetabular surfaces, sphericity and cartilage thickness. J. Biomech. 14:253-260, 1981.

${ }^{25}$ Sledge, S. L. Microfracture techniques in the treatment of osteochondral injuries. Clin. Sports Med. 20:365-377, 2001.

${ }^{26}$ Soltz, M. A., and G. A. Ateshian. Experimental verification and theoretical prediction of cartilage interstitial fluid pressurization at an impermeable contact interface in confined compression. J. Biomech. 31:927-934, 1998.

${ }^{27}$ Swartz, M. A., and M. E. Fleury. Interstitial flow and its effects in soft tissues. Annu. Rev. Biomed. Eng. 9:229-256, 2007.

${ }^{28}$ Treppo, S., A. Grodzinsky, E. Quan, E. Frank, E. Bombard, and D. Breslau. Arthroscopic impedance probe to detect cartilage degeneration. U.S. Patent \#6856834, Massachusetts Institute of Technology, 2005.

${ }^{29}$ Wang, D., C. G. Williams, F. Yang, and J. H. Elisseeff. Enhancing the tissue-biomaterial interface: tissue-initiated integration of biomaterials. Adv. Funct. Mater. 14:1152$1159,2004$.

${ }^{30}$ Wong, B. L., W. C. Bae, J. Chun, K. R. Gratz, M. Lotz, and R. L. Sah. Biomechanics of cartilage articulation: effects of lubrication and degeneration on shear deformation. Arthritis Rheum. 58:2065-2074, 2008.

${ }^{31}$ Wong, B. L., and R. L. Sah. Effect of a focal articular defect on cartilage deformation during patello-femoral articulation. J. Orthop. Res. 28:1554-1561, 2010.

${ }^{32}$ Xie, L., A. S. Lin, R. E. Guldberg, and M. E. Levenston. Nondestructive assessment of SGAG content and distribution in normal and degraded rat articular cartilage via EPIC-muCT. Osteoarthr. Cartil. 18(1):65-72, 2009. 